\title{
Crystal growth, structural perfection, phase transition, optical, and etching studies of doped glycine phosphite ferroelectric single crystals
}

\author{
R. Perumal ${ }^{\mathrm{a}, *}$, S. Moorthy Babu ${ }^{\mathrm{a}}, \mathrm{G}$. Bhagavannarayana ${ }^{\mathrm{b}}$ \\ a Crystal Growth Centre, AnnaUniversity Chennai, Chennai 600025, India \\ b Crystal Growth and Crystallography Section, National Physical Laboratory, CSIR, Dr. K.S. Krishnan Marg, New Delhi 110012, India
}

\begin{abstract}
A B S T R A C T
Glycine phosphite (GPI) single crystal was doped with complexing agent allylthiourea, rare-earth, and metal ions (cesium and iron). The dopant concentrations were varied between 1 and $3 \mathrm{~mol} \%$. Influence of the dopants on the structural perfection was studied using the X-ray diffraction method. Effects of the dopants on the phase transition temperature were examined using the impedance analytical method. The optical properties of the as-grown crystals were studied using a UV spectrophotometer. The surface morphology and the presenting defects were also studied by the chemical etching method and the obtained results have been presented.
\end{abstract}

\section{Introduction}

The crystal of many inorganic derivatives of amino acids provides excellent crystals such as triglycine sulphate, L-arginine phosphate accepted for the fabrication of devices [1]. Glycine phosphite (GPI), one of the hydrogen bonded ferroelectric single crystals, belongs to this family of crystals. The GPI crystal undergoes a continuous ferroelectric-paraelectric phase transition at $224.7 \mathrm{~K}$ [2]. In the paraelectric phase, GPI belongs to monoclinic crystal structure with $P_{21 / a}$ space group when it turns to the ferroelectric phase, the crystal system remains the same but the phase group changes from the centrosymmetric to the non-centrosymmetric $P_{21}$ phase group [3]. Many practical devices using ferroelectrics require a phase transition temperature above or closer to the room temperature. The value of the phase transition temperature when it shifts towards to the room temperature increases the utility of GPI single crystals for the fabrication of the devices. In the GPI crystal, ferroelectrics appear due to the ordering of protons in the interphosphite hydrogen bonds. Hence, a large isotopic effect can be expected by isotopic substitution for hydrogen in the GPI crystal which drastically increases the phase transition temperature above the room temperature. This improves its suitability for high sensitive pyroelectric sensors capable of working at room temperature [4].
Subsitutional or interstitial impurities in the host lattice leads to significant changes in the properties of the pure TGS crystals [5-7]. The Doping of cobalt (II) phosphate in TGSP crystal resulted in good quality crystals with a stable domain structure [8]. Doped TGSP crystals with amino acids such as L-alanine (L-ATGSP) and L-asparagine (ASP-TGSP), were investigated for further improvement in their pyroelectric property [9]. The phosphoric acid doped TGS resulted in an increase in the pyroelectric coefficient than pure TGS and a shift in the phase transition temperature was observed [10]. Crystal perfection and performance of ZTS was considerably improved by the organic dopant [11]. Previously glycine phosphate doped GPI [12], urea and thiourea doped GPI [13,14] have been reported. In this direction, attempts were made to dope GPI crystal with an organic complexing agent and various metal ions to improve the phase transition temperature. Doping efforts on the growth aspects, structural perfection, phase transition temperature, and optical properties were studied by conducting various characterization techniques. Presenting defects of the grown crystals were examined using the chemical etching method and the obtained results have been presented.

\section{Experimental procedure}

The dopants were taken in the form of nitride (lanthanum), carbonate (cesium), chloride (iron) and synthesized salts (allylthiourea) were added directly to the saturated solution. The materials were synthesized using the appropriate addition of dopants such as rare-earth ion (lanthanum, $3 \mathrm{~mol} \%$ ), organic complexing agent (allylthiourea, $10 \mathrm{wt} \%$ ), alkali metal ion (cesium, $1 \mathrm{~mol} \%$ ) and transition metal ion (iron, $1 \mathrm{~mol} \%$ ) in different ratios (molar and weight percentage). The recrystallisation process was adapted for further purification of the starting materials. Low temperature solution growth method was used for the crystal growth experiments and the growth runs were preformed in an aqueous solution. The grown crystals were named as LGPI, AGPI, CGPI and FGPI for lanthanum-, allylthiourea-, cesium- 

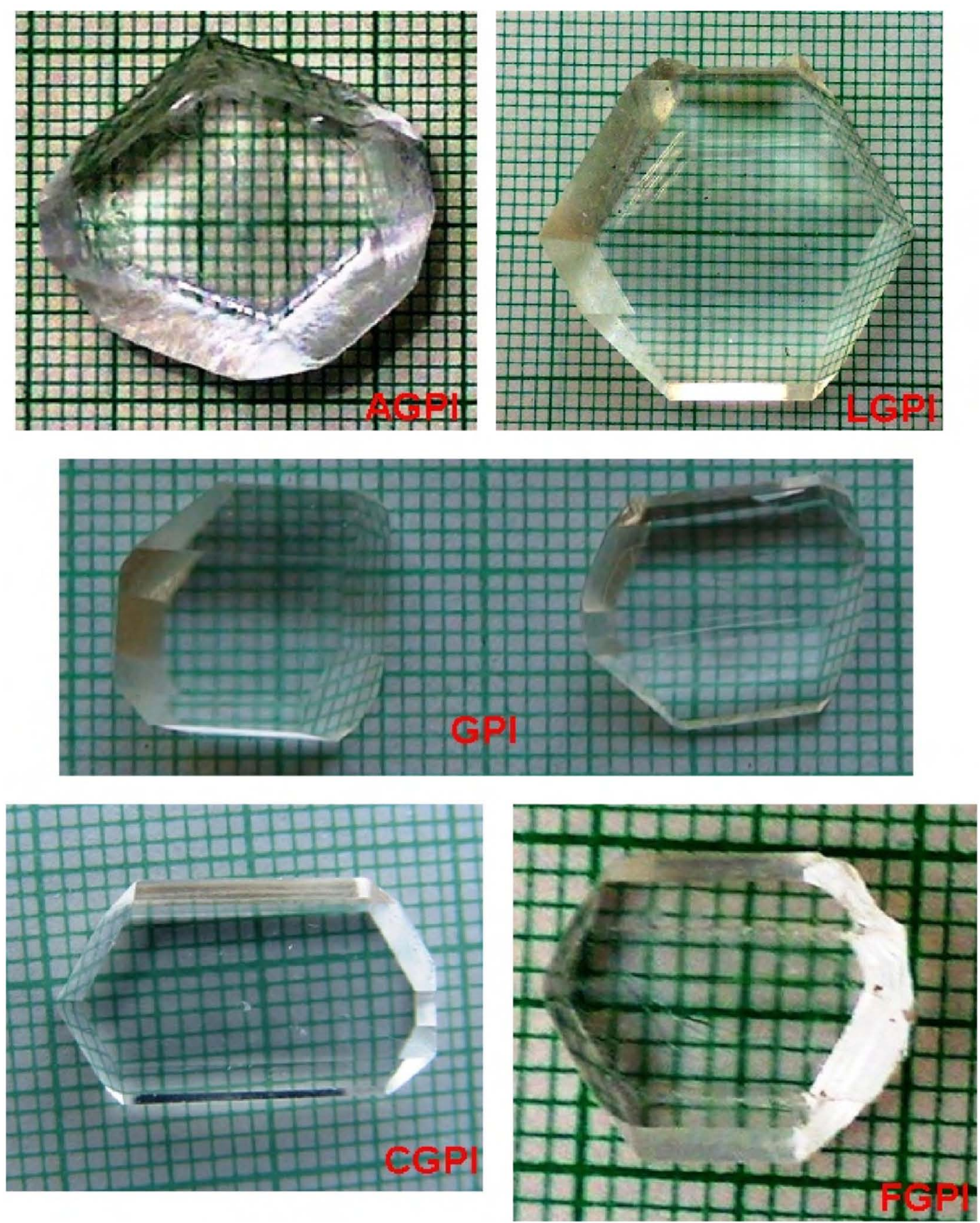

Fig. 1. Doped GPI crystals grown by slow solvent evaporation technique.

and iron-doped GPI crystals, respectively. The structural perfection of the as-grown crystal specimens were identified with the X-ray rocking curve using a high resolution multicrystal X-ray diffractometer. The ferroelectric behaviors of the grown specimens were analyzed using HP impedance gain-phase analyzer; model HP in the liquid $\mathrm{N}_{2}$ ambient. Optical transmittance spectra were recorded using a Shimadzu UV-vis spectrophotometer. The etching microstructures were examined using an optical microscope (Leitz metallux-II) in the reflection mode.

\section{Results and discussions}

Good quality single crystals of pure and doped GPI were grown by controlled solvent evaporation technique and the grown crystals are shown in Fig. 1. It was anticipated that the dopants may go to the interstitial sites and not disturb the crystal lattice. Crystal perfection analysis is an important study to evaluate the device performance of the grown crystals. The rocking curves for the all doped GPI crystals are shown in Fig. 2. A similar experimental condition
[15] was adapted for recording the rocking curves. The full width at half maximum (FWHM) values for AGPI, LGPI, CGPI, and FGPI specimens were $16,30,15$, and 34 arc $s$, respectively which is more than that corresponding to the pure GPI crystal [15]. The broadening of diffraction curve revealed the incorporation of dopants into the crystalline matrix. The lower FWHM values of each main peak shows that the grown crystalline perfection is reasonably good and the all grown specimens were free from structural grain boundaries except the FGPI crystal. In LGPI, the scattered intensity was much more in the negative direction in comparison with the positive direction which was the reason for the asymmetry nature of the obtained diffraction curve. This feature clearly indicates that the crystal contains predominantly vacancy type of defects than interstitial defects [16]. The lattice around these defects undergoes tensile stress and the lattice parameter ' $d$ ' (interplanar spacing) increases which leads to more scattered intensity. Generally, this 

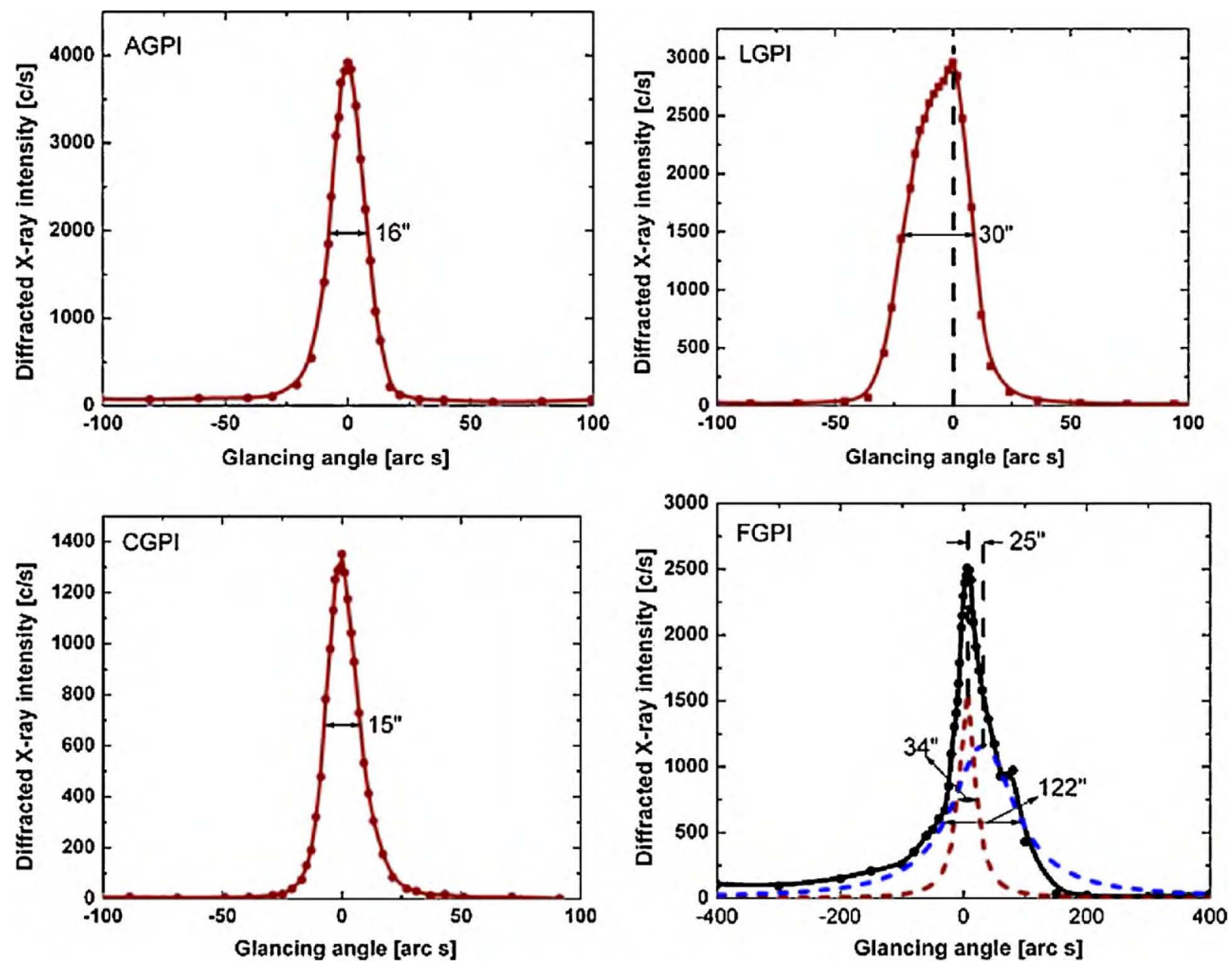

Fig. 2. Diffraction curves recorded for doped GPI single crystal for $(020)$ diffracting planes.

type of a defect may be arises due to the fast growth. Because, the growth rates differ for the various planes of the grown crystals. It is obvious that the FGPI crystal diffraction curve contains an additional peak, which is 25 arc s away from the main peak, depicts the presence of an internal structural at very low angle (tilt angle

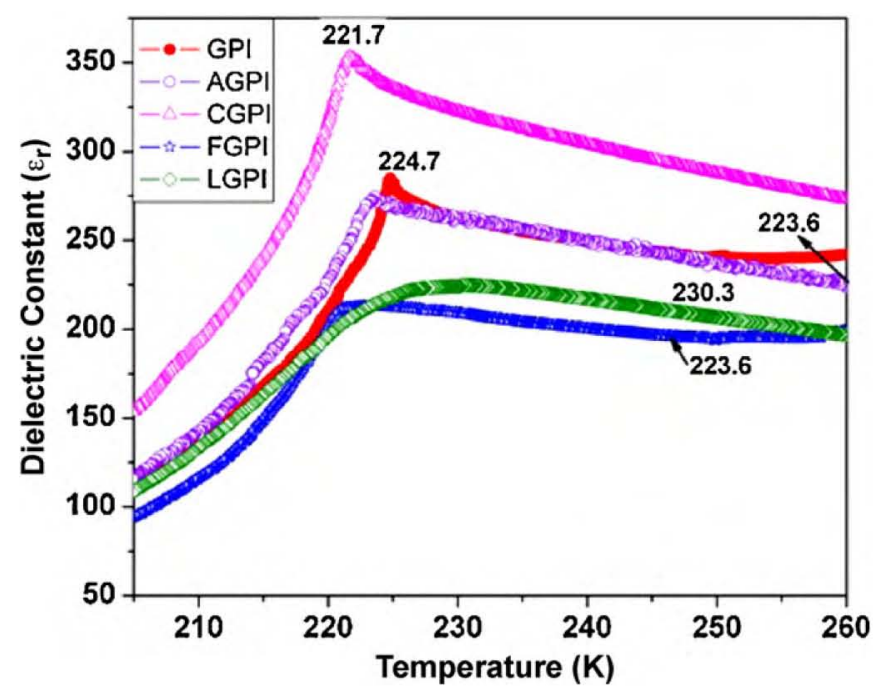

$\alpha<1$ arc min), the grain boundary [17] whose tilt angle (misorientation angle between the two crystalline regions on both sides of the structural grain boundary) is 25 s from its adjoining main crystal block. The FWHM of the main crystalline block and the very low angle grain regions were 34 and 122 arcs. The relatively low angular spread of around 400 arc s of the diffraction curve and the low FWHM value of the main peak reveals that the crystalline per-

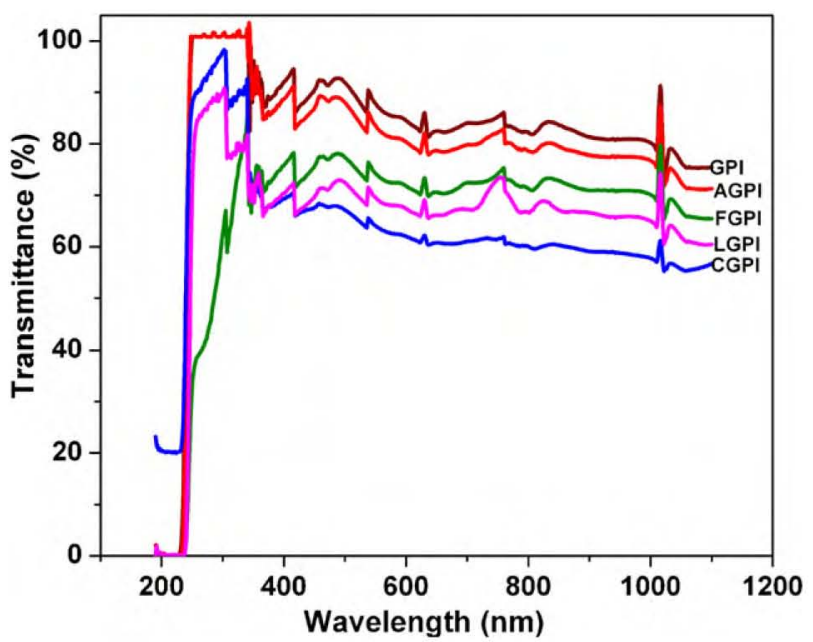

Fig. 4. Optical transmittance spectrum of doped GPI single crystals. 
(a)

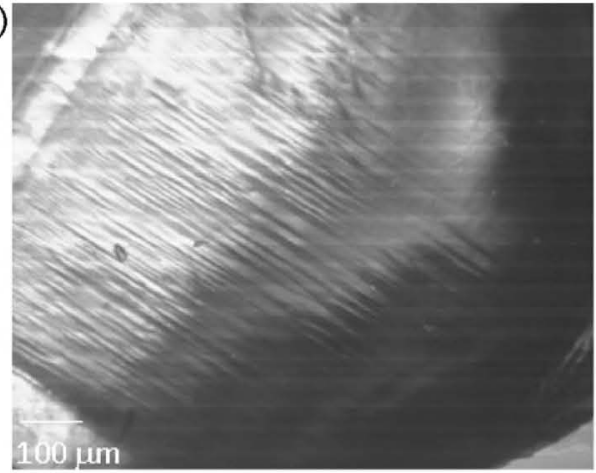

(c)

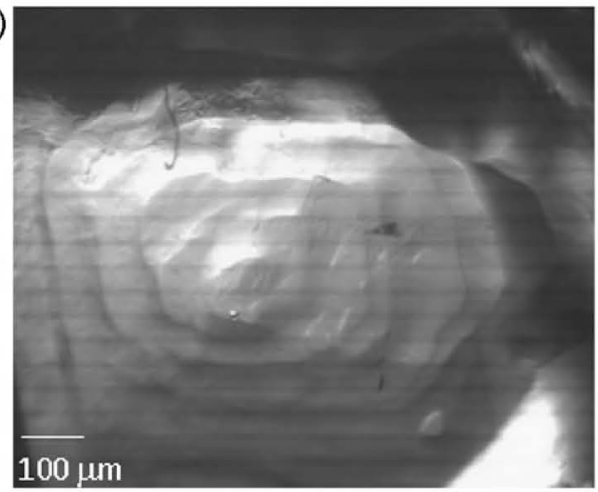

(b)

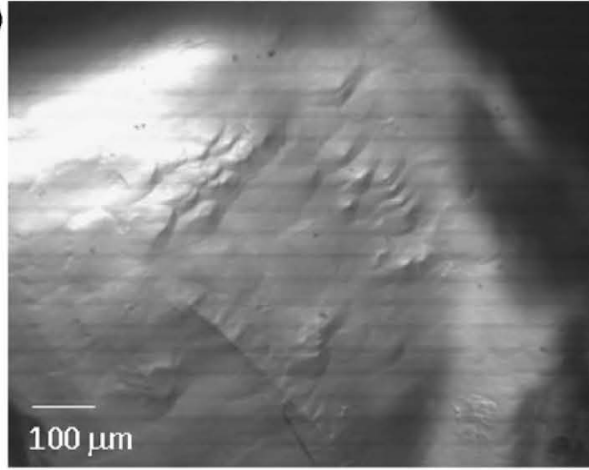

(d)

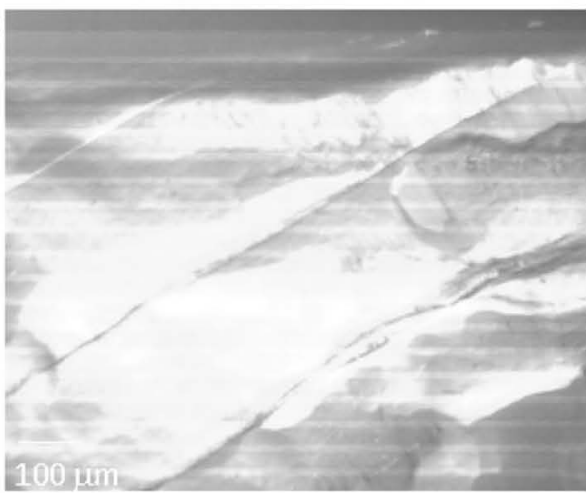

Fig. 5. Etching micrographs of doped GPI crystals.

fection was reasonably good. However, the presenting defects had lower density for AGPI, LGPI, and CGPI, but the concentration was high, for FGPI and hence this led to the structural grain boundaries which could affect the performance of the devices based on such FGPI crystals.

Dielectric studies are an essential parameter for all ferroelectric crystals to study their exact phase transition temperature. The effects of dopants on the phase transition temperature were identified through dielectric measurements. The measurement details provided in the previous paper [15] and the resultant curves are shown in Fig. 3. The dopants shifted the phase transition temperature slightly, but the shift was not considerable compared with the deuteration effect [4]. The dielectric permittivity value increased and reached a maximum at the phase transition temperature after that permittivity decreased continuously to a constant value. Dipole ordering appeared at the phase transition temperature due to the domain nature of the as-grown crystalline specimen. The sharp peak at the phase transition temperature reveals that the grown crystal was of the continuous phase transition nature. It was noted that the permittivity value of CGPI was higher in comparison with pure GPI and this value was low for LGPI and FGPI. But, in the case of AGPI crystal this value was nearly the same as pure GPI. Among the dopants, the rare-earth influenced (LGPI) effectively the phase transition temperature compared with others. The optical transmittance spectra of pure and doped GPI crystals are shown in Fig. 4. The grown crystals are completely transparent in the entire UV region without any significant absorbance and all the grown crystals have a cut-off around at $250 \mathrm{~nm}$ and the dopants do not influence the absorbance edge. All the crystals have a large transmittance window which is very important and a most desirable property for a variety of optical applications.

Chemical etching is one of the simple and the powerful tool to analyze the presenting defect in the as-grown crystalline surfaces [18]. Dislocations easily appear in crystals, especially in the initial stages of their growth. In the present investigation, chemical etch- ing was carried out using Millipore water (resistivity $18.2 \mathrm{M} \Omega$ ) as etchant at room temperature. Once the damaged surface layer was removed by means of etching, a fresh surface appeared, which in turn gave clear etch pits. The etched samples were immediately examined and their microstructures are shown in Fig. 5. The rectilinear elongated etch patterns (Fig. 5a) and linear steps (Fig. 5d) were seen in the etching patterns which revealed two-dimensional (2D) layer growth mechanisms. The well-defined pyramidal shape hillocks (Fig. 5b) were observed on the dislocation sites which perhaps were due to the result of some sort of overgrowth in the etching media or due to the protection of the surface against etching. Growth spirals (Fig. 5c) observed which have been formed on the surface of crystals grown at low supersaturation, such a spiral in fact corresponds to a group of dislocations.

\section{Conclusions}

Good quality single crystals of pure and doped glycine phosphite ferroelectric single crystal were grown using low temperature solution growth method. The structural perfection study of the grown crystals ascertained the good crystal quality. Rare-earth ions effectively influence the phase transition temperature in comparison with the other dopants. No modifications were observed from the optical studies due to the dopants. The appearances of the welldefined etch pits shows that the grown crystals were affected by selective type of dislocations.

\section{References}

[1] M.E. Lines, A.M. Glass, Principles and Applications of Ferroelectrics and Related Materials, Clarendon Press, Oxford, 1977.

[2] S. Dacko, Z. Czapla, J. Baran, M. Drozd, Phys. Lett. A 223 (1996) 217-220.

[3] M. Therese, A. Pouchot, Cryst. Struct. Commun. C C49 (1993) 815-818.

[4] J. Baran, M.S. Sledz, R. Jakubas, G. Bator, Phys. Rev. B 55 (1997) 169-172.

[5] R. Muralidharan, R. Mohankumar, P.M. Ushasree, R. Jayavel, P. Ramasamy, J. Cryst. Growth 234 (2002) 545-550. 
[6] J. Stankowska, T. Jasinski, Acta Phys. Pol. A 1 (1986) 559-563.

[7] E. Mihaylova, S.T. Stoyanov, Phys. Status Solidi. A 154 (1996) 797-802.

[8] L. Prokopova, J. Novotny, Z. Micka, V. Malina, Cryst. Res. Technol. 36 (2001) $1189-1195$.

[9] O.W. Wang, C.S. Fang, Cryst. Res. Technol. 27 (1992) 245-251.

[10] G. Ravi, G. Arunmozhi, S. Anbukumar, P. Ramasamy, Ferroelectrics 174 (1995) 241-247.

[11] G. Bhagavannarayana, S. Parthiban, S. Meenakshisundaram, J. Appl. Cryst. 39 (2006) 784-790.

[12] V.K. Yarmarkin, S.N. Popov, S.G. Shulman, V.V. Lk, Ferroelectrics 320 (2005) 91-97.
[13] S. Kalainathan, M. Beatrice Margaret, Mater. Sci. Eng. B 120 (2005) 190-193.

[14] R. Ezhil Vizhi, S. Kalainathan, G. Bhagavannarayana, Cryst. Res. Technol. 43 (2008) $778-782$.

[15] R. Perumal, K. Senthil Kumar, S. Moorthy Babu, G. Bhagavannarayana, J. Alloys Compd. 490 (2009) 342-349.

[16] R. Perumal, S. Moorthy Babu, Curr. Appl. Phys. 10 (2009) 858-865.

[17] G. Bhagavannarayana, R.V. Ananthamurthy, G.C. Budakoti, B. Kumar, K.S. Bartwal, J. Appl. Cryst. 38 (2005) 768-771.

[18] K. Sangwal, Etching of Crystals: Theory, Experiment and Application, North Holland Physics Publishing, Amsterdam, The Netherlands, 1987. 